\title{
Humic acid-stabilized superparamagnetic maghemite nanoparticles: surface charge and embryotoxicity evaluation
}

\author{
A. E. Goldt ${ }^{1}$, A. Yu. Polyakov ${ }^{2 *}$, T. A. Sorkina ${ }^{3}$, A. L. Dubov ${ }^{4,5}$, G. A. Davidova ${ }^{6}$, I. I. Selezneva ${ }^{6}$, \\ Y. V. Maximov ${ }^{7}$, I. A. Presnyakov ${ }^{5}$, N. Yu. Polyakova $^{8}$, E. A. Goodilin ${ }^{2,4,5}$, I. V. Perminova ${ }^{5}$ \\ ${ }^{1}$ Skolkovo Institute of Science and Technology, Moscow, Russia \\ ${ }^{2}$ Kurnakov Institute of General and Inorganic Chemistry, Russian Academy of Sciences, Moscow 119991, Russia \\ ${ }^{3}$ Science \& Technology Department, Management Company RUSNANO, LLC, Moscow, Russia \\ ${ }^{4}$ Department of Materials Science, Lomonosov Moscow State University, Moscow 119991, Russia \\ ${ }^{5}$ Department of Chemistry, Lomonosov Moscow State University, Moscow 119991, Russia \\ ${ }^{6}$ Institute of Theoretical \& Experimental Biophysics of Russian Academy of Sciences, Pushchino 142290, Russia \\ ${ }^{7}$ Semenov Institute of Chemical Physics of Russian Academy of Sciences, Moscow 119991, Russia \\ ${ }^{8}$ I. M. Sechenov First Moscow State Medical University of the Ministry of Health of the Russian Federation \\ (Sechenov University), Moscow, Russia
}

*a.yu.polyakov@gmail.com

\begin{abstract}
Superparamagnetic iron oxide $\gamma-\mathrm{Fe}_{2} \mathrm{O}_{3}$ (maghemite) nanoparticles (SPION) encapsulated into water-soluble microspheres of rock salt were synthesized via a new aerosol spray pyrolysis procedure. Humic acids (HA) were employed to stabilize the aqueous suspensions of $\gamma-\mathrm{Fe}_{2} \mathrm{O}_{3}$ nanoparticles released upon dissolution of the $\mathrm{NaCl}$ matrix. The effect of $\mathrm{HA}$ on the surface charge of maghemite-based colloids was studied in $\mathrm{pH}$ range of $3-10$. Humic polyanions compensate positive charges on a hydrated $\gamma-\mathrm{Fe}_{2} \mathrm{O}_{3}$ surface resulting in strongly negative $\zeta$-potential $(<-40 \mathrm{mV})$ of colloid even in acidic environment. In neutral and alkaline environment, $\zeta$-potential of maghemite-based colloid drops below $-55 \mathrm{mV}$; thus, HA should effectively stabilize the nanoparticle colloid over the whole $\mathrm{pH}$ range studied. Meanwhile, bare maghemite SPION at $\mathrm{pH} 3-6$ have $\zeta$-potential in the $+20 \mathrm{mV}$ to $-20 \mathrm{mV}$ range (isoelectric point at $\mathrm{pH} 4.35$ ), which is insufficient for electrostatic stabilization of the suspensions. The absence of embryotoxicity of HA-stabilized nanoparticles was demonstrated.
\end{abstract}

Keywords: small superparamagnetic iron oxide nanoparticles (SPION), humic acids, magnetic fluids, colloidal properties, embryotoxicity, biomedicine.

Received: 20 January 2019

Revised: 31 January 2019

\section{Introduction}

Superparamagnetic iron oxide nanoparticles (SPIONs) with a size less than $5 \mathrm{~nm}$ have attracted growing attention as emerging nanomaterials for biomedical applications, including magnetic resonance imaging (MRI), drug delivery and theranostics, due to their high biocompatibility, chemical stability, tunable surface features, prolonged blood circulation time due to the reduced phagocytosis by macrophages and $\mathrm{T}_{1}$-shortening effect (unlike larger iron oxide nanoparticles) in MRI [1-4].

To achieve enhanced colloidal stability and versatility of biomedical applications, numerous approaches have been developed for the surface modification of SPIONs employing the specifically designed synthetic ligands [1, 5]. Meanwhile, the cheap and effective natural stabilizers for the SPIONs are still of high demand. It was previously demonstrated that the humic acids (HA), i.e. the natural organic matter originating from biochemical and microbiological transformations of organic materials under environmental conditions, can efficiently stabilize iron oxide nanoparticles due to the numerous highly developed branches with irregularly located organic functional groups [6-11]. However, the colloidal stability of the resulting core-shell organic-inorganic nanomaterials at different $\mathrm{pH}$ values was not characterized, while it is crucial for biomedical applications of the stabilized SPIONs. Additionally, the toxicity of these nanomaterials were evaluated on NCTC clone L929 cells [6], but never studied on the embryos, while the absence of embryotoxicity is an important criterion applicable to the new biomedical agents.

Here, we report the effect of humic acids on the surface charge of water-dispersed ultrasmall superparamagnetic $\gamma-\mathrm{Fe}_{2} \mathrm{O}_{3}$ nanoparticles at different $\mathrm{pH}$ values, as well as embryotoxicity evaluation of these stabilized SPIONs. 


\section{Experimental section}

\subsection{Synthesis}

$\mathrm{Fe}\left(\mathrm{NO}_{3}\right)_{3} \cdot 9 \mathrm{H}_{2} \mathrm{O}, \mathrm{NaCl}$ and urea of analytical purity grade were purchased from Sigma-Aldrich. Leonardite humic acids (HA) were isolated from the commercially available potassium humate (Powhumus, Humintech Ltd., Germany) [12]. To prepare $100 \mathrm{mg} / \mathrm{l} \mathrm{HA}$ solution, a weight of the solid sample was dissolved first in a few $\mathrm{ml}$ of $1.0 \mathrm{M} \mathrm{NaOH}$ upon sonication for $20 \mathrm{~min}$ at room temperature, diluted by deionized water (Milli-Q, Millipore), and adjusted to $\mathrm{pH} 7.0$ using $0.1 \mathrm{M} \mathrm{HCl}$.

Maghemite nanoparticles incorporated into the $\mathrm{NaCl}$ microspheres were synthesized according to the aerosol spray pyrolysis (ASP) procedure described elsewhere [13]. In brief, dry $\mathrm{NaCl}$ was added to $0.25 \mathrm{M}$ aqueous $\mathrm{Fe}\left(\mathrm{NO}_{3}\right)_{3}$ to achieve final molar ratio of $\gamma-\mathrm{Fe}_{2} \mathrm{O}_{3}$ to $\mathrm{NaCl}$ of $1: 10$. Urea was also added to the solution to enhance the combustion in the hot zone and yield finer nanoparticles. The obtained solution was atomized using an ultrasonic nebulizer (resonant frequency of $1.7 \mathrm{MHz}, 0.5-5$ micron solution droplets). The aerosol stream was injected into a horizontal quartz reactor $\left(20 \mathrm{~mm}\right.$ inner diameter, $900 \mathrm{~mm}$ length) pre-heated to $650{ }^{\circ} \mathrm{C}$. The flow rate of air used as a carrier gas was $10 \mathrm{~L} / \mathrm{min}$, resulting in a transfer of the spray through the hot zone during ca. $5 \mathrm{sec}$. The resulting powders were collected at a surface of a microporous glass fiber filter after the aerosol has been transported and transformed in the hot zone.

To prepare a magnetic fluid, the obtained microspheres were dispersed in $100 \mathrm{mg} / \mathrm{l} \mathrm{HA}$ solution followed by ultrasonic treatment for $20 \mathrm{~min}$. Concentration of iron oxide was $43 \mathrm{mg} / 1$ (30 mg/l Fe(III)), which corresponds to $200 \mathrm{mg} / \mathrm{l}$ of salt-maghemite composite. The salt from the composite also provides $157 \mathrm{mg} / 1(2.7 \mathrm{mmol} / \mathrm{l}) \mathrm{NaCl}$ concentration in the resulting colloid. For the further transmission electron microscopy and Mössbauer spectroscopy studies, the suspension of HA-stabilized SPIONs was sedimented by 10 min centrifugation at $7000 \mathrm{rpm}$ and dried in ambient air.

\subsection{Physicochemical characterization}

Scanning electron microscopy (SEM) images were obtained using a Leo Supra 50 VP microscope (Carl Zeiss) at accelerating voltage of $5 \mathrm{kV}$. Transmission electron microscopy (TEM) images were obtained using a Hitachi H-8100 transmission electron microscope (accelerating voltage of $200 \mathrm{kV}$ ) to investigate morphology and size of nanoparticles.

Mössbauer spectroscopy was used to study SPIONs at 77 and $300 \mathrm{~K}$ using a constant-acceleration WissEl spectrometer (Germany) equipped with a krypton proportional detector, a $\gamma$-radiation source of ${ }^{57} \mathrm{Co}$ in a rhodium matrix, and a Janis helium cryostat (model CCS-850). Chemical shifts were referred to metallic $\alpha$-iron. The spectra were fitted using the least square minimization procedure by the standard software.

$\zeta$-Potential values were determined using Zetasizer Nano ZS (Malvern Instruments) at $25{ }^{\circ} \mathrm{C}$. Standard folded capillary $\zeta$-cells were employed. The $\mathrm{pH}$ of the nanoparticle colloids was adjusted using $0.1 \mathrm{M} \mathrm{HCl} \mathrm{and} 0.1 \mathrm{M}$ $\mathrm{NaOH}$ to study the changes of $\zeta$-potential in the $\mathrm{pH}$ range of $3-10$ (starting from $\mathrm{pH} 3$ and going to the higher $\mathrm{pHs}$ ).

\subsection{Embryotoxicity}

The embryotoxicity of the obtained SPIONs was assessed using in vitro mice embryo growth tests. To culture the embryos, 16 cultural liquid (Sigma, $\mathrm{pH} 7.0-7.3,37{ }^{\circ} \mathrm{C}, 5 \% \mathrm{CO}_{2}$ ) was used. In total, 40 mice embryos were tested in this study. $\gamma-\mathrm{Fe}_{2} \mathrm{O}_{3}$ and HA-stabilized $\gamma-\mathrm{Fe}_{2} \mathrm{O}_{3}$ were added to the embryos-containing liquid at the ratio of 1:10. Growing embryos were monitored up to the blastocyst stage in the control group using optical microscopy (Axiovert 200, Zeiss, Germany) for counting a number of blastocysts and characterization of their anomalies.

\section{Results and discussion}

ASP is a known effective technique for continuous and scalable synthesis of iron oxide nanoparticles and allows successful preparation of the metastable superparamagnetic phases, like $\gamma-\mathrm{Fe}_{2} \mathrm{O}_{3}$ [13]. The morphology of the composite obtained by ASP is known to be dependent on the concentration of the sprayed precursor solution, flow velocity (i.e. the duration of the spray transfer through the hot zone) and the furnace temperature [13]. The particles obtained in this work upon $5 \mathrm{~s}$ transfer of ultrasonic fog through a hot zone $\left(650{ }^{\circ} \mathrm{C}\right)$ consisted of hollow $0.5-2 \mu \mathrm{m} \mathrm{NaCl}$ microspheres formed from submicron solution droplets loaded with ultrasmall iron oxide nanoparticles (Fig. 1a). Dissolution of the obtained microspheres in aqueous humic acid solution results in a stable sol of iron oxide nanoparticles. To disaggregate the SPIONs more effectively, an ultrasonic treatment has been applied resulting in separate $2-5 \mathrm{~nm}$ nanoparticles (Fig. 1c), as observed by TEM of the centrifuged SPION-HA colloid (Fig. 1b). Note that the nanoparticle sizes calculated from TEM images $(2-5 \mathrm{~nm} ; 3.5 \pm 0.8 \mathrm{~nm}$ mean 
size) are significantly below the hydrodynamic diameter $(145 \pm 60 \mathrm{~nm})$ reported for the same SPION-HA colloids elsewhere [6]. This supports the previous hypothesis that SPIONs not only adsorb the humic substances but also penetrate into the branched structure of the conglomerates of humic acid molecules and become assembled there [6,7]. A simplified illustration of this process is given on Fig. 2. It should be noted that a similar assembling effect was found for the synthetic dendrimers and $\mathrm{Fe}_{3} \mathrm{O}_{4}$ nanoparticles [14].
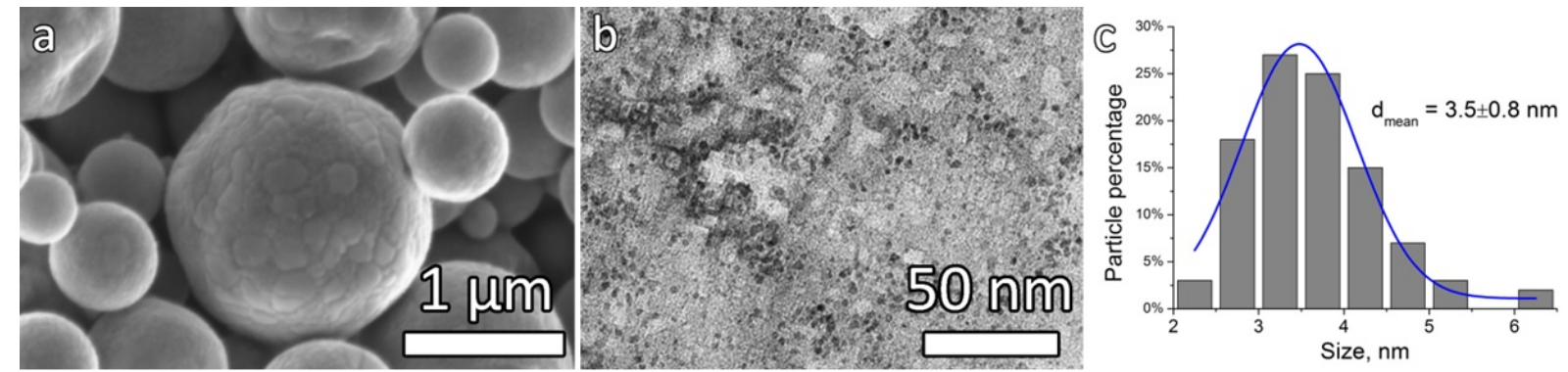

FIG. 1. (a) SEM image of the SPION-NaCl microspheres obtained by the ASP method at $650{ }^{\circ} \mathrm{C}$. (b) TEM image of the SPION sol obtained by dispersion/dissolution of SPION-NaCl microspheres in the aqueous humic acid solution and sedimented by $10 \mathrm{~min}$ centrifugation at $7000 \mathrm{rpm}$. Note the amorphous HA mass in which the SPIONs are distributed. (c) Size distribution of the SPIONs in suspension as observed by TEM
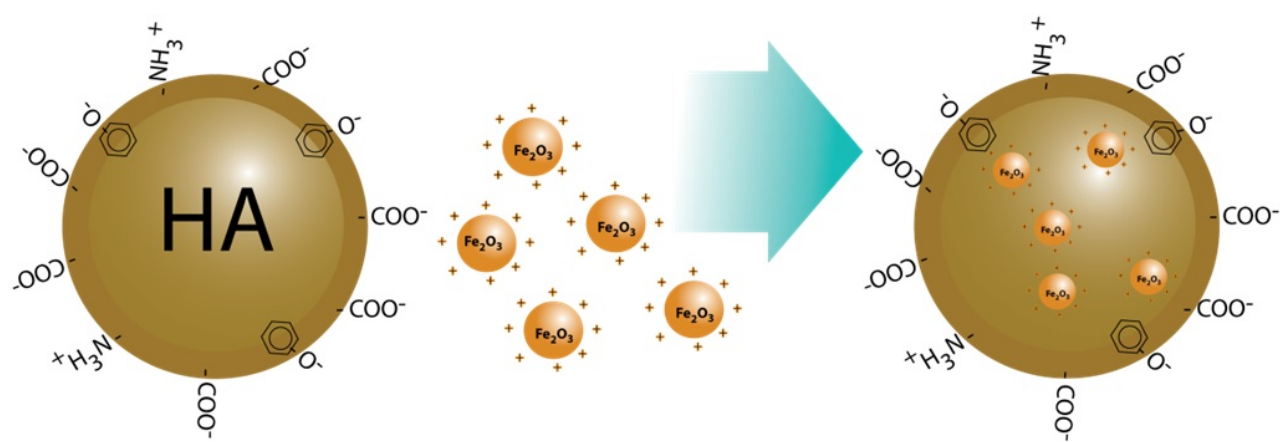

FIG. 2. A simplified scheme for stabilization of $\gamma-\mathrm{Fe}_{2} \mathrm{O}_{3}$ nanoparticles by conglomerates (possibly, micelles) of humic acid molecules

The superparamagnetic behavior of dried HA-stabilized $\gamma-\mathrm{Fe}_{2} \mathrm{O}_{3}$ nanoparticles was confirmed using Mössbauer spectroscopy at $300 \mathrm{~K}$ and $77 \mathrm{~K}$, which is also a powerful tool to study the iron oxide speciation $[8,15]$. The characteristic paramagnetic signal (doublet) observed at room temperature (Fig. 3a) switches to hyperfine structure lines, which were fitted by 3 sextet components (Fig. 3b, Table 1).

The Mossbauer spectrum of maghemite nanoparticles at $77 \mathrm{~K}$ is known to be rather complicated and is usually fitted by multiple components [16], ascribed to the different $\mathrm{Fe}^{+3}$ positions in the $\left(\mathrm{Fe}^{+3}{ }_{8}\right)_{A}\left[\mathrm{Fe}^{+3}{ }_{40 / 3} \square_{8 / 3}\right]_{B} \mathrm{O}_{32}$ spinel phase ( $\square$ represents the vacancies of the octahedral sites), impact of the surface states [17], etc. In our

TABLE 1. Mössbauer parameters of $\gamma-\mathrm{Fe}_{2} \mathrm{O}_{3}$ particles stabilized by $\mathrm{HA}$ at $77 \mathrm{~K}$ [ $\delta$ is the isomer shift relative to $\alpha-\mathrm{Fe}, \varepsilon$ is the quadrupole splitting, $\Gamma$ is the line width, and $H_{i n}$ is the internal magnetic field $(T)$ ]

\begin{tabular}{|c|c|c|c|c|c|}
\hline \multirow{2}{*}{ Component } & $\delta$ & $\varepsilon$ & $\Gamma$ & $\begin{array}{c}H_{\text {in }}, \\
\pm 0.5 T\end{array}$ & $\begin{array}{c}\text { Relative content (\%), } \\
\pm 1 \%\end{array}$ \\
\cline { 2 - 4 } & \multicolumn{2}{|c|}{ $\pm 0.03 \mathrm{~mm} / \mathrm{s}$} & \pm 0.5 & 33 \\
\hline \hline$\gamma-\mathrm{Fe}_{2} \mathrm{O}_{3}-1$ & 0.41 & 0.00 & 0.78 & 45.4 & 36 \\
\hline$\gamma-\mathrm{Fe}_{2} \mathrm{O}_{3}-2$ & 0.42 & 0.00 & 0.78 & 48.7 & 46 \\
\hline$\gamma-\mathrm{Fe}_{2} \mathrm{O}_{3}-3$ & 0.43 & 0.00 & 0.78 & 39.9 & 21 \\
\hline
\end{tabular}



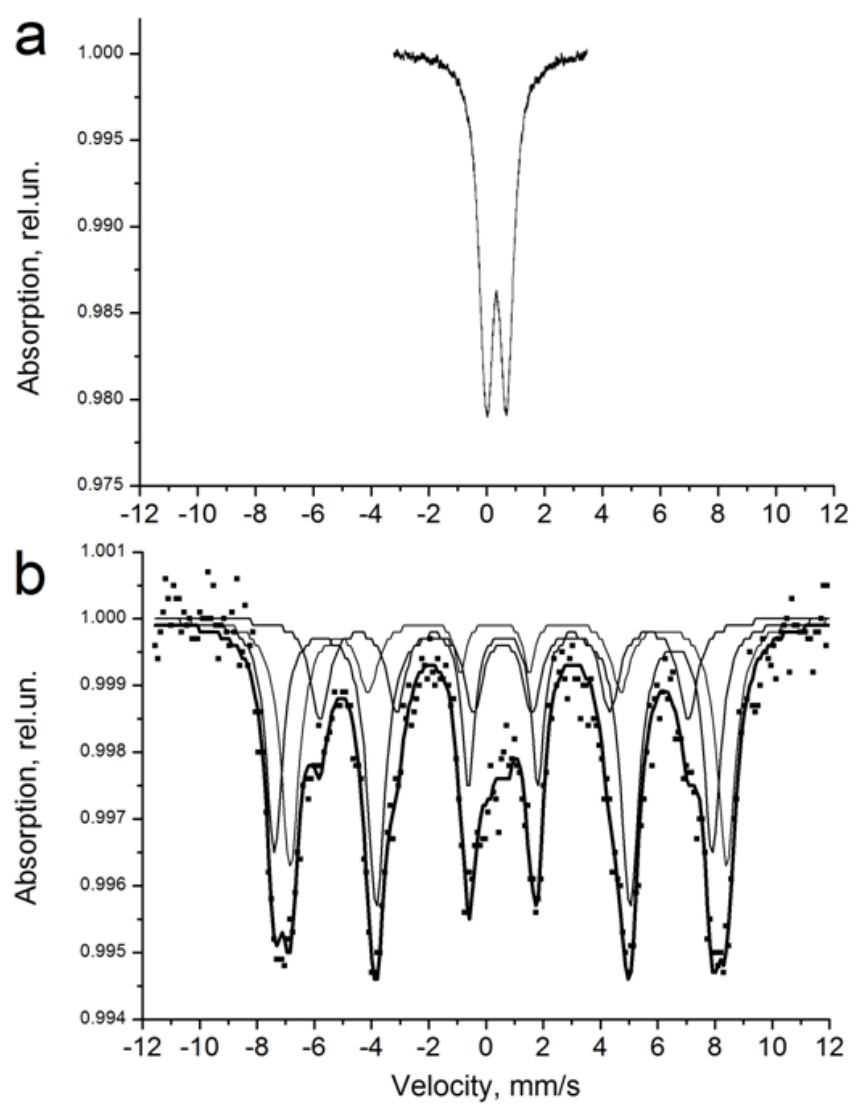

FIG. 3. ${ }^{57} \mathrm{Fe}$ Mössbauer spectra of the dried SPION-HA suspension at $300 \mathrm{~K}$ (a) and $77 \mathrm{~K}$ (b)

previous Mössbauer study, the low temperature $(63-90 \mathrm{~K})$ spectra of the dry $\gamma-\mathrm{Fe}_{2} \mathrm{O}_{3}-\mathrm{NaCl}$ nanocomposite were fitted by two sextets with the internal magnetic fields $\left(H_{i n}\right)$ of $43-50 T$ [13]. Therefore, the additional component with the lower $H_{\text {in }}\left(\delta=0.43 \pm 0.03 \mathrm{~mm} / \mathrm{s}, \varepsilon=0.00 \pm 0.03 \mathrm{~mm} / \mathrm{s}\right.$ and $\left.H_{\text {in }}=39.9 \pm 0.5 T\right)$ can be related to the interaction of HA with the surface of magnetic phase.

When dispersed in aqueous medium, iron oxide nanoparticles are hydrated and their surface is enriched with $\mathrm{Fe}-\mathrm{OH}$ sites demonstrating an amphoteric behavior, i.e. reacting with $\mathrm{H}^{+}$or $\mathrm{OH}^{-}$ions from dissolved acids and bases (depending on $\mathrm{pH}$ value) and producing the positive $\left(\mathrm{Fe}-\mathrm{OH}_{2}^{+}\right)$or negative $\left(\mathrm{Fe}-\mathrm{O}^{-}\right)$charges, respectively [9]. The changes of the surface charge affect the electrostatic repulsion between the nanoparticles and thus, the overall stability of the colloid according to the DLVO theory $[9,18,19]$. Here, we employed $\zeta$-potential measurements to characterize the surface charge of $\gamma-\mathrm{Fe}_{2} \mathrm{O}_{3}$ nanoparticles and their conglomerates with $\mathrm{HA}$ at $\mathrm{pH}$ range of $3-10$ (Fig. 4). At $\mathrm{pH} \mathrm{3,} \mathrm{the} \zeta$-potential of the bare SPIONs (released upon dissolution of $\mathrm{NaCl}$ component of the ASP-produced microspheres) is $+18.5 \mathrm{mV}$ and decreases monotonically with the pH growth over the whole studied range. The nanoparticles remain positively charged below $\mathrm{pH} 4.35$ and have negative charges at higher $\mathrm{pH}$ values. The $\mathrm{pH} 4.35$ at which the surface charge of $\gamma-\mathrm{Fe}_{2} \mathrm{O}_{3}$ switch from positive (predominance of $\mathrm{Fe}-\mathrm{OH}_{2}^{+}$ groups) to negative (predominance of $\mathrm{Fe}_{-} \mathrm{O}^{-}$groups) can be considered as the isoelectric point (IEP) for the obtained maghemite nanoparticles. The observed IEP for the ASP-synthesized $\gamma-\mathrm{Fe}_{2} \mathrm{O}_{3}$ is significantly lower than that reported for the maghemite nanoparticles synthesized by co-precipitation $\left(\mathrm{pH}_{\mathrm{IEP}}=6.6[20]\right)$. Generally, the particles with a $\zeta$-potential higher than $+30 \mathrm{mV}$ or lower than $-30 \mathrm{mV}$ are considered to be electrostatically stable in colloids; at lower $\zeta$-potential values, the particles are prone to agglomeration [21,22]. Note that the $\zeta$-potential of non-stabilized ASP-synthesized $\gamma-\mathrm{Fe}_{2} \mathrm{O}_{3}$ nanoparticles is below these threshold values in acidic medium.

The presence of $100 \mathrm{mg} / \mathrm{L} \mathrm{HA}$ drastically changes the surface charge of the SPION colloid. Even at low $\mathrm{pH}$ values $\zeta$-potential becomes strongly negative $(-40 \mathrm{mV}$ at $\mathrm{pH} 3)$ and drops below $-55 \mathrm{mV}$ at $\mathrm{pH}>7$. It seems that a high amount of the carboxylate-rich humic polyanions adsorbed on the surface of the SPIONs (and entrapping them as discussed above) leads to neutralization $\left(\mathrm{Fe}-\mathrm{OH}_{2}^{+}+\mathrm{Hum}_{-} \mathrm{COO}^{-} \leftrightarrow \mathrm{Fe}-\mathrm{OOC}-\mathrm{Hum}+\mathrm{H}_{2} \mathrm{O}\right)$ and then compensation of positive charges on the iron oxide surface even in acidic medium. A similar effect of humic and fulvic acids on the iron oxide surface charge was previously reported for the magnetite [9] and hematite [23-25] 


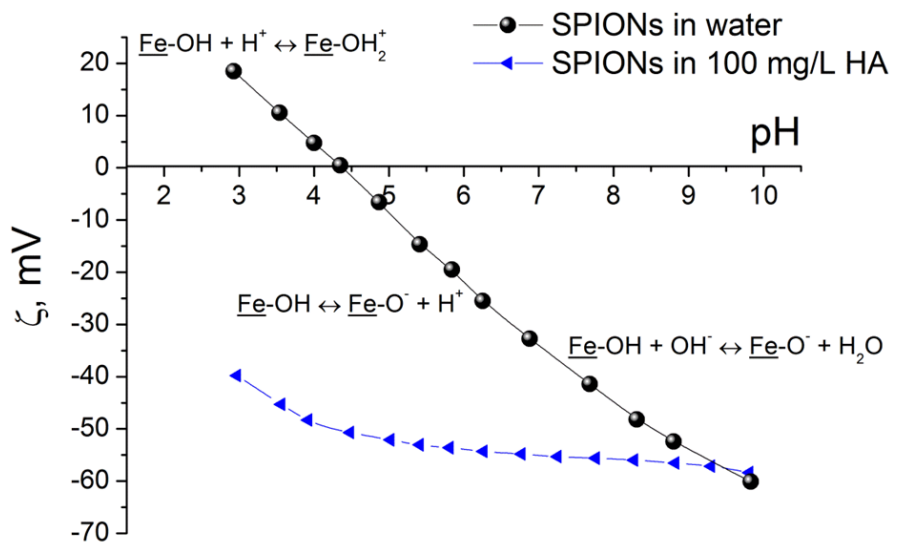

FIG. 4. $\zeta$-potential of SPIONs as a function of $\mathrm{pH}$ in the absence and in the presence of $100 \mathrm{mg} / \mathrm{L}$ $\mathrm{HA}$ solution at room temperature. The concentration of SPION-NaCl composite is $200 \mathrm{mg} / \mathrm{L}$, which corresponds to $30 \mathrm{mg} / \mathrm{L}$ on $\mathrm{Fe}(\mathrm{III})$ basis

particles. The repulsion of the strong negative charges provided by humic polyanions leads to stabilization of the SPION sol over the whole $\mathrm{pH}$ range studied.

The influence of nanoparticles on the development of embryos seems to be quite important to avoid embryotoxic effects of new medical agents [26-28]. Our analysis has evidently demonstrated (Fig. 5) that HA-coated $\gamma-\mathrm{Fe}_{2} \mathrm{O}_{3}$ has no negative effects on in vitro growth of mice embryos. Moreover, SPION-added embryos gave about $10 \%$ surplus of healthy blastocysts with respect to the control group. These data support our previous cytotoxicity study, which demonstrated no toxicity of the same HA-stabilized $\gamma-\mathrm{Fe}_{2} \mathrm{O}_{3}$ nanoparticles with respect to the NCTC clone L929 cells [6], and provide additional justification for a future possibility of HA-coated SPION applications in biomedicine.

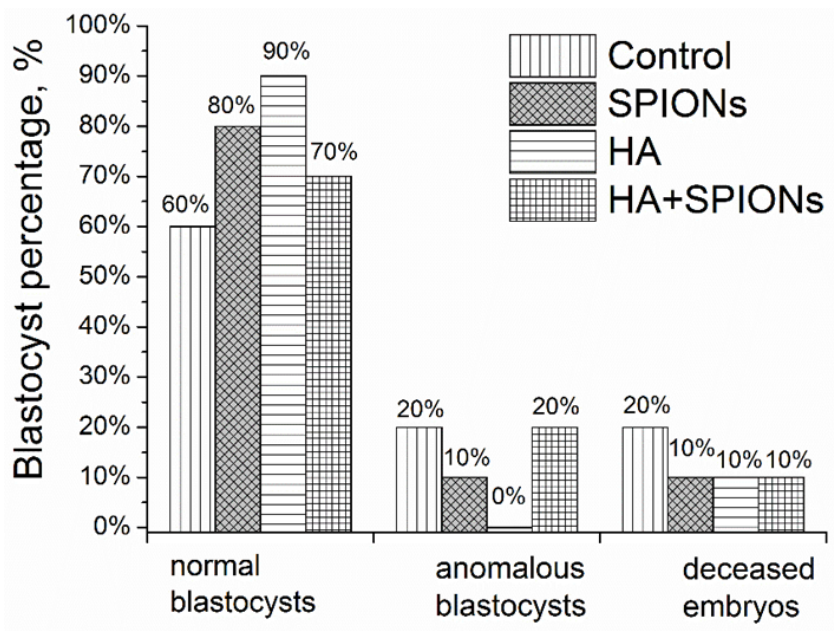

FIG. 5. In vitro influence of nude and HA-stabilized SPIONs (released from the $\gamma-\mathrm{Fe}_{2} \mathrm{O}_{3}-\mathrm{NaCl}$ nanocomposite) on the viability of mice 2-cell embryos

\section{Conclusions}

Humic acids show a significant effect on the surface charge of ASP-synthesized ultrasmall $(2-5 \mathrm{~nm})$ superparamagnetic $\gamma-\mathrm{Fe}_{2} \mathrm{O}_{3}$ nanoparticles in aqueous suspensions. While non-modified $\gamma-\mathrm{Fe}_{2} \mathrm{O}_{3}$ nanoparticles have $\zeta$-potential within $+20 \mathrm{mV}$ to $-20 \mathrm{mV}$ range at $\mathrm{pH} 3-6$ (isoelectric point at $\mathrm{pH} 4.35$ ), humic substances shift the $\zeta$-potential towards much lower values $(<-40 \mathrm{mV})$ required for the effective electrostatic stabilization of the colloids. Importantly, the in vitro studies revealed no embryotoxic effect of the designed HA-stabilized sols against the mice 2-cell embryos. These data strengthen the role of HA as an effective biocompatible stabilizing agent for magnetic fluids in possible biomedical applications. 


\section{Acknowledgements}

The work was carried out within the State Assignment on Fundamental Research to the Kurnakov Institute of General and Inorganic Chemistry.

\section{References}

[1] Song C., Sun W., Xiao Y., Shi X. Ultrasmall iron oxide nanoparticles: synthesis, surface modification, assembly, and biomedical applications. Drug Discov. Today, 2019, 24 (3), P. 835-844.

[2] Weissleder R., Elizondo G., et al. Ultrasmall superparamagnetic iron oxide: characterization of a new class of contrast agents for MR imaging. Radiology, 1990, 175 (2), P. 489-493.

[3] Rui Y.-P., Liang B., et al. Ultra-large-scale production of ultrasmall superparamagnetic iron oxide nanoparticles for $\mathrm{T}_{1}$-weighted MRI. RSC Adv., 2016, 6 (27), P. 22575-22585.

[4] Emashova N.A., Kudryashov V.E., et al. Quo vadis, worldwide nanoindustry. Nanotechnologies Russ., 2016, 11 (3-4), P. 117-127.

[5] Dimarco M., Guilbert I., et al. Colloidal stability of ultrasmall superparamagnetic iron oxide (USPIO) particles with different coatings. Int. J. Pharm., 2007, 331 (2), P. 197-203.

[6] Chekanova A.E., Sorkina T.A., et al. New environmental nontoxic agents for the preparation of core-shell magnetic nanoparticles. Mendeleev Commun., 2009, 19 (2), P. 72-74.

[7] Polyakov A.Y., Goldt A.E., et al. Constrained growth of anisotropic magnetic $\delta$-FeOOH nanoparticles in the presence of humic substances. CrystEngComm, 2012, 14 (23), P. 8097-8102.

[8] Polyakov A.Y., Sorkina T.A., et al. Mössbauer spectroscopy of frozen solutions as a stepwise control tool in preparation of biocompatible humic-stabilized feroxyhyte nanoparticles. Hyperfine Interact., 2013, 219 (1-3), P. 113-120.

[9] Illés E., Tombácz E. The effect of humic acid adsorption on pH-dependent surface charging and aggregation of magnetite nanoparticles. J. Colloid Interface Sci., 2006, 295 (1), P. 115-123.

[10] Demangeat E., Pédrot M., et al. Colloidal and chemical stabilities of iron oxide nanoparticles in aqueous solutions: the interplay of structural, chemical and environmental drivers. Environ. Sci. Nano, 2018, 5 (4), P. 992-1001.

[11] Kulikova N.A., Polyakov A.Y., et al. Key Roles of Size and Crystallinity of Nanosized Iron Hydr(oxides) Stabilized by Humic Substances in Iron Bioavailability to Plants. J. Agric. Food Chem., 2017, 65 (51), P. 11157-11169.

[12] Perminova I.V., Kovalenko A.N., et al. Design of Quinonoid-Enriched Humic Materials with Enhanced Redox Properties. Environ. Sci. Technol., 2005, 39 (21), P. 8518-8524.

[13] Chekanova A.E., Dubov A.L., et al. Soluble microcapsules for non-toxic magnetic fluids. Mendeleev Commun., 2009, 19 (1), P. 4-6.

[14] Yang J., Luo Y., et al. Conjugation of Iron Oxide Nanoparticles with RGD-Modified Dendrimers for Targeted Tumor MR Imaging. ACS Appl. Mater. Interfaces, 2015, 7 (9), P. 5420-5428.

[15] Kirillova S.A., Almjasheva O.V., Panchuk V.V., Semenov V.G. Solid-phase interaction in $\mathrm{ZrO}_{2}-\mathrm{Fe}_{2} \mathrm{O}_{3}$ nanocrystalline system. Nanosyst. Phys. Chem. Math., 2018, 9 (6), P. 763-769.

[16] Ramos Guivar J.A., Bustamante A., et al. Mössbauer study of intermediate superparamagnetic relaxation of maghemite $\left(\gamma-\mathrm{Fe}_{2} \mathrm{O}_{3}\right)$ nanoparticles. Hyperfine Interact., 2014, 224 (1-3), P. 89-97.

[17] Zakharova I.N., Shipilin M.A., Alekseev V.P., Shipilin A.M. Mössbauer study of maghemite nanoparticles. Tech. Phys. Lett., 2012, 38 (1), P. 55-58.

[18] Polyakov A.Y., Lebedev V.A., et al. Non-classical growth of water-redispersible spheroidal gold nanoparticles assisted by leonardite humate. CrystEngComm, 2017, 19 (5), P. 876-886.

[19] Israelachvili J. Intermolecular and Surface Forces. Academic Press, Elsevier, Burlington, 2011, 710 p.

[20] Nurdin I., Syarofi R., Satriananda. The Effect of pH and Time on The Stability of Superparamagnetic Maghemite Nanoparticle Suspensions. MATEC Web Conf., 2016, 39, 01001, DOI: 10.1051/matecconf/20163901001.

[21] Sikora A., Bartczak D., et al. A systematic comparison of different techniques to determine the zeta potential of silica nanoparticles in biological medium. Anal. Methods, 2015, 7 (23), P. 9835-9843.

[22] Večeř M., Pospíšil J. Stability and Rheology of Aqueous Suspensions. Procedia Eng., 2012, 42, P. 1720-1725.

[23] Amal R., Raper J.A., Waite T.D. Effect of fulvic acid adsorption on the aggregation kinetics and structure of hematite particles. J. Colloid Interface Sci., 1992, 151 (1), P. 244-257.

[24] Verrall K.E., Warwick P., Fairhurst A.J. Application of the Schulze-Hardy rule to haematite and haematite/humate colloid stability. Colloids Surfaces A Physicochem. Eng. Asp., 1999, 150 (1-3), P. 261-273.

[25] Ramos-Tejada M.M., Ontiveros A., Viota J.L., Durán J.D.G. Interfacial and rheological properties of humic acid/hematite suspensions. $J$. Colloid Interface Sci., 2003, 268 (1), P. 85-95.

[26] Peng G., He Y., et al. Differential effects of metal oxide nanoparticles on zebrafish embryos and developing larvae. Environ. Sci. Nano, 2018, 5 (5), P. 1200-1207.

[27] Patel S., Jana S., et al. Toxicity evaluation of magnetic iron oxide nanoparticles reveals neuronal loss in chicken embryo. Drug Chem. Toxicol., 2019, 42 (1), P. 1-8.

[28] Magro M., De Liguoro M., et al. The surface reactivity of iron oxide nanoparticles as a potential hazard for aquatic environments: A study on Daphnia magna adults and embryos. Sci. Rep., 2018, 8 (1), 13017. 